\title{
Contributions to metric-topological localization and mapping in mobile robotics *
}

\author{
Eduardo Jesús Fernández Moral \\ Departmento de Ingeniería de Sistemas y Automática, Universidad de Málaga, Spain \\ Advisor: Javier González-Jiménez \\ PhD thesis defended 27th October 2014, Universidad de Málaga
}

Received 23rd March 2015; accepted 27th July 2015

\begin{abstract}
This thesis addresses the problem of localization and mapping in mobile robotics. The ability of a robot to build a map of an unknown environment from sensory information is required to perform self-localization and autonomous navigation, as a necessary condition to carry out more complex tasks. This problem has been widely investigated in the last decades, but the solutions presented still present important limitations, mainly they have to cope with large scale and dynamic environments, and to work in a wider range of conditions and scenarios. In this context, this thesis takes a step forward towards highly efficient localization and mapping.

A first contribution of this work is a new mapping strategy that presents two key features: the lightweight representation of world metric information, and the organization of this metric map into a topological structure that allows efficient localization and map optimization. Regarding the first issue, a map is proposed based on planar patches which are extracted from range or RGB-D images. This plane-based map (PbMap) is particularly well suited for indoor scenarios, and has the advantage of being a very compact and still a descriptive representation which is useful to perform real-time place recognition and loop closure. These operations are based on matching planar features taking into account their geometric relationships. On the other hand, the abstraction of metric information is necessary to deal with large scale SLAM and with navigation in complex environments. For that, we propose to structure the map in a metric-topological structure which is dynamically organized upon the sensor observations.

Also, a simultaneous localization and mapping (SLAM) system employing an omnidirectional RGB-D device which combines several structured-light sensors (Asus Xtion Pro Live) is presented (see fig 1). This device allows the quick construction of rich models of the environment at a relative low cost in comparison with previous alternatives. Our SLAM approach is based on a hierarchical structure of keyframes with a low level layer of metric information and several topological layers intended for large scale SLAM and navigation. This SLAM solution, which makes use of the metric-topological representation mentioned above, works at video frame rate obtaining highly consistent maps. Future research is expected on metric-topological-semantic mapping from the new sensor and the SLAM system presented here.
\end{abstract}

This thesis is publicly available in http://riuma.uma.es/xmlui/handle/10630/9931

Correspondence to: <efernandezmoral@gmail.com>

Recommended for acceptance by Jorge Bernal

DOI http://dx.doi.org/10.5565/rev/elcvia.738

ELCVIA ISSN:1577-5097

Published by Computer Vision Center / Universitat Autònoma de Barcelona, Barcelona, Spain 


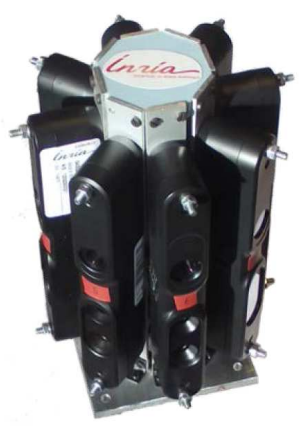

(a)

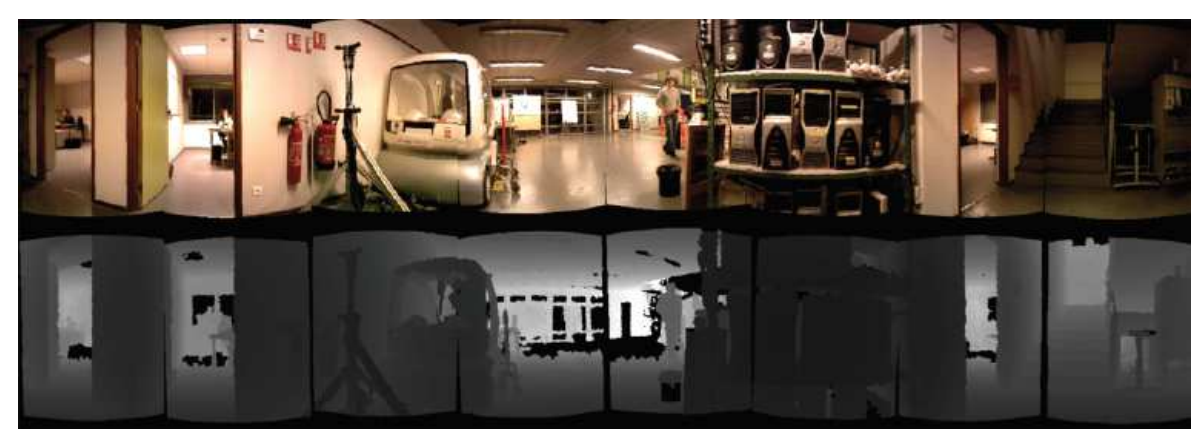

(b)

Figure 1: a) Omnidirectional RGB-D camera rig and b) an image captured with it.

Finally, an extrinsic calibration technique is proposed to obtain the relative poses of a combination of 3D range sensors, like those employed in the omnidirectional RGB-D device in fig. 10 The calibration is computed from the observation of planar surfaces of a structured environment in a fast, easy and robust way, presenting qualitative and quantitative advantages with respect to previous approaches. This technique is extended to calibrate any combination of range sensors, including 2D and 3D range sensors, in any configuration. The calibration of such sets of sensors is interesting not only for mobile robots, but also for autonomous cars.

A list of the publications produced from this thesis is presented below.

\section{Bibliography}

1. E. Fernández-Moral, J. González-Jiménez and V. Arévalo, "Extrinsic calibration of 2D laser rangefinders from perpendicular plane observations" The International Journal of Robotics Research. Sage Publications (2015). DOI: 10.1177/0278364915580683

2. E. Fernández-Moral, V. Arévalo and J. González-Jiménez, "Hybrid metric -topological mapping for large scale monocular SLAM", Informatics in Control, Automation and Robotics, 2015. Pages 217-232.

3. E. Fernández-Moral, V. Arévalo and J. González-Jiménez, "Extrinsic calibration of a set of 2D laser rangefinderss", Proceedings of the IEEE International Conference on Robotics and Automation (ICRA), 2015.

4. E. Fernández-Moral, J. González-Jiménez, P. Rives and V. Arévalo, "Extrinsic calibration of a set of range cameras in 5 seconds without any pattern", Proceedings of the IEEE/RSJ International Conference on Intelligent Robots and Systems (IROS), 2014.

5. E. Fernández-Moral, V. Arévalo and J. González-Jiménez, "A compact planar-patch descriptor based on color", Proceedings of the International Conference on Informatics in Control, Automation and Robotics (ICINCO), 2014.

6. T. Gokhool, M. Meilland, P. Rives and E. Fernández-Moral, "Dense RGB-D map building from spherical RGB-D images", Proceedings of the 9th International Conference on Computer Vision, Imaging and Computer Graphics Theory and Applications (VISAPP), 2014.

7. E. Fernández-Moral, V. Arévalo and J. González-Jiménez, "Creating metric-topological maps for largescale monocular SLAM", Proceedings of the International Conference on Informatics in Control, Automation and Robotics (ICINCO), 2013. 
8. E. Fernández-Moral, W. Mayol-Cuevas, V. Arévalo and J. González-Jiménez, "Fast place recognition with plane-based maps", Proceedings of the IEEE International Conference on Robotics and Automation (ICRA), 2013. 